\title{
Dynamics of spinning particle pairs in a single-layer complex plasma crystal
}

\author{
V. Nosenko, ${ }^{1,2}, *$ S. K. Zhdanov, ${ }^{1}$ H. M. Thomas, ${ }^{1}$ J. Carmona-Reyes, ${ }^{2}$ and T. W. Hyde ${ }^{2}$ \\ ${ }^{1}$ Institut für Materialphysik im Weltraum, Deutsches Zentrum für Luft- und Raumfahrt (DLR), D-82234 Weßling, Germany \\ ${ }^{2}$ Center for Astrophysics, Space Physics, and Engineering Research, \\ Baylor University, Waco, Texas 76798-7310, USA
}

(Dated: July 20, 2017)

\begin{abstract}
Spontaneous formation of spinning pairs of particles, or torsions was studied in a single-layer complex plasma crystal by reducing the discharge power at constant neutral gas pressure. At higher gas pressures, torsions spontaneously formed below a certain power threshold. Further reduction of the discharge power led to the formation of multiple torsions. However, at lower gas pressures the torsion formation was preceded by the mode-coupling instability (MCI). The crystal dynamics were studied with the help of the fluctuation spectra of crystal particles' in-plane velocities. Surprisingly, the spectra of the crystal with torsions and MCI are rather similar and contain "hot spots" at similar locations on the $(k, \omega)$ plane, despite very different appearances of the respective particle trajectories. The torsion rotation speed was close (slightly below) to the maximum frequency of the in-plane compressional mode. When multiple torsions formed, their rotation speeds were distributed in a narrow range slightly below the maximum frequency.
\end{abstract}

PACS numbers: $52.27 . \mathrm{Lw}, 52.27 . \mathrm{Gr}$

Introduction. - A complex plasma is a suspension of charged microparticles in a weakly ionized gas [1]. If the interaction between particles is strong enough, they can self-organize into ordered structures, e.g., plasma crystals. In the presence of gravity, monodisperse microparticles can form single-layer, or two-dimensional (2D) plasma crystals. They are popular model systems to study various phenomena such as waves [2-5], phase transitions [6-8], and transport phenomena [9-13], in real time and at the level of individual particles. However, care should be taken to clearly separate the generic and plasma-related phenomena which are peculiar to plasma crystals [14]. The latter may be, e.g., mode-coupling instability (MCI) [15-18], channeling of upstream extra particles [19], or particle pairing [20,21]. Vertical pairs of particles can form in a single-layer plasma crystal spontaneously [20] or after a sudden drop of the neutral gas pressure [21]. Pairs of particles which were spinning around their axis were recently observed in Ref. [22]. Such spinning pairs, or torsions can form in a single-layer complex plasma crystal if the vertical confinement becomes sufficiently weak. A theoretical model of a spinning particle pair based on the plasma wake (ion focus) effect was proposed in Ref. [22]. According to this model, torsions are driven by the nonreciprocal interactions between dust particles mediated by the plasma wakes. The energy input is balanced by the neutral gas friction. Such spinning pairs of particles are different from the instability described in Refs. [23, 24] in the following ways: (1) isolated pairs of particles spontaneously appear in a singlelayer plasma crystal vs. instability in an aligned bilayer of particles, (2) particle pairs perform coherent rotation vs. random oscillations, (3) their appearance is triggered by the reduction of the discharge power vs. instability triggered by the reduction of the gas pressure.

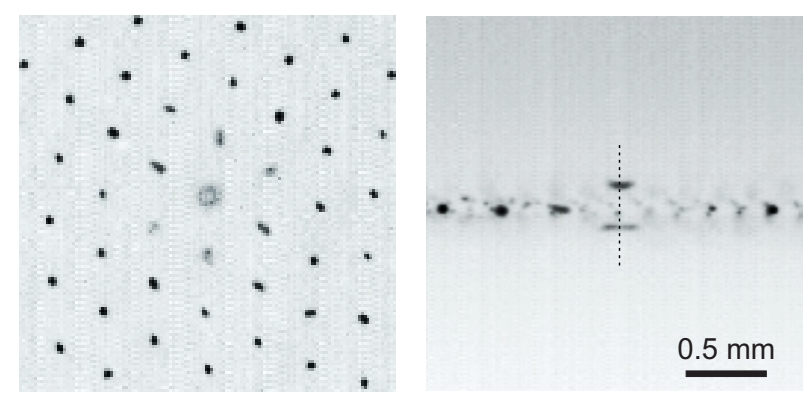

FIG. 1. Spinning pair of particles in a single-layer plasma crystal. Left panel shows the top view, right panel the side view. The particles spin around the vertical axis shown by the dashed line. Notice that the lattice cell containing the pair is enlarged by $\approx 10 \%$. 90 frames from the video are superimposed, the resulting image is inverted and its brightness and contrast adjusted. Experimental parameters were the following: argon pressure $p_{\mathrm{Ar}}=17.4 \mathrm{~Pa}$, discharge power $P_{\mathrm{rf}}=7 \mathrm{~W}$.

In the experiment of Ref. [22], microparticles were suspended in argon plasma at the (relatively high) pressure of $20.9 \mathrm{~Pa}$. In this case, particle dynamics were strongly damped due to friction created by the neutral gas, making it impossible to measure the phonon spectra of the plasma crystal.

In the present work, we systematically study torsion formation using lower gas pressures down to $12 \mathrm{~Pa}$, in order to better reveal the accompanying particle dynamics.

Experimental method. - The experiments were performed in CASPER's modified Gaseous Electronics Conference (GEC) rf reference cell [22, 25]. Plasma was produced by a capacitively coupled rf discharge in argon. The lower disk-shaped electrode was rf-powered; a grounded ring-shaped electrode above it and the cham- 


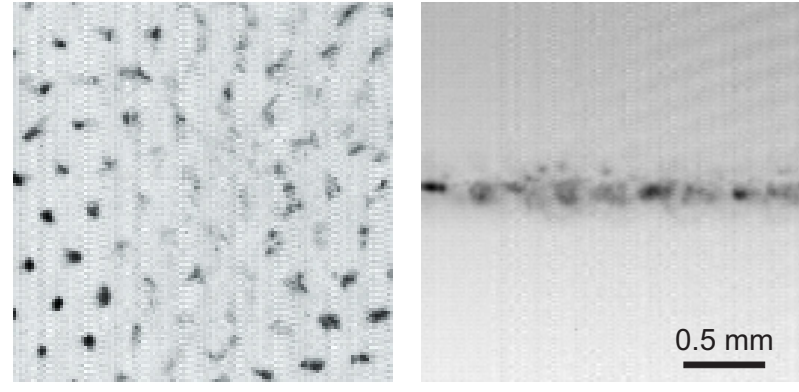

FIG. 2. Mode-coupling instability in a single-layer plasma crystal. Left panel shows the top view, right panel the side view. 90 frames from the video are superimposed, the resulting image is inverted and its brightness and contrast adjusted. Experimental parameters were the following: argon pressure $p_{\mathrm{Ar}}=12 \mathrm{~Pa}$, discharge power $P_{\mathrm{rf}}=15 \mathrm{~W}$.

ber walls served as the other electrode. Melamineformaldehyde microspheres with a diameter of $8.9 \pm$ $0.09 \mu \mathrm{m}$ were suspended as a single layer in the plasma sheath above the lower rf elecrode. The plasma crystal was "purified" using the standard procedure [19]. The argon pressure $p_{\mathrm{Ar}}$ was then set to either 19.3, 17.4, or $12 \mathrm{~Pa}$ and the forward discharge power $P_{\mathrm{rf}}$ was stepwise reduced from 40 to $1 \mathrm{~W}$. This resulted in a weakening of the vertical confinement for the particles, see, e.g., Ref. [16], and eventual departure from the single-layer structure particle suspension. The same particle suspension was used for all combinations of $p_{\mathrm{Ar}}$ and $P_{\mathrm{rf}}$.

In each experimental run, top-view and side-view video of the particle suspension were recorded. Two Photron FASTCAM PCI 1024 cameras with K-2 Infinity lenses (with a focal length of about $30 \mathrm{~cm}$ ) operating at 125 frames per second were used for recording. A StingRay $100 \mathrm{~mW}$ red laser was used for illuminating the layer of particles. Another laser (with a maximum power of $80 \mathrm{~mW}$ and wavelength of $640 \mathrm{~nm}$ ) was used to illuminate a vertical slice of the particle suspension for the side view. We selected the vertical slice that contained the feature of interest, e.g., a torsion [26].

Particle coordinates and velocities were then calculated in each frame using a moment method [27]. Particle velocity power spectra $V^{2}(k, f)$ (fluctuation spectra for longitudinal mode) were calculated using the following method [2]. First, a uniform square portion of the plasma crystal sized $9.34 \times 9.34 \mathrm{~mm}^{2}$ and containing the feature of interest (e.g., a torsion) was chosen and its orientation was determined from its static structure factor. Second, the wave propagation direction $\theta=0^{\circ}$ was chosen. (This corresponds to the angle of $30^{\circ}$ between the wave vector $\mathbf{k}$ and the direction to a nearest neighbor). Third, the particle velocity component parallel to $\mathbf{k}$ was spatially averaged within 128 rectangular bins elongated orthogonally to k. Fourth, the Fourier transform of the averaged particle velocity was then computed in the space and
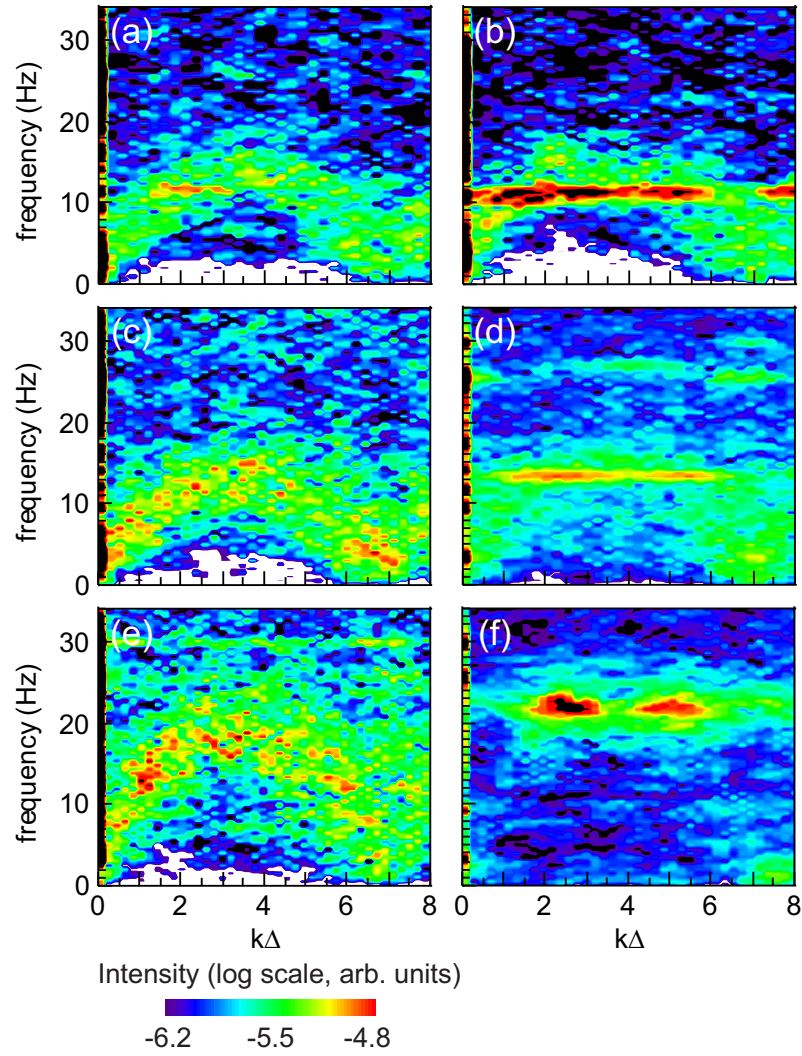

FIG. 3. Fluctuation spectra of the particles' in-plane velocities within a crystal (longitudinal mode). Spectral intensity is color-coded as indicated, except for panels (d) and (f), where the scale is from -6.2 to -3.6 and from -5.4 to -2.8 , respectively. The wave propagation direction was $\theta=0^{\circ}$ (see the text for more details). The wave number $k$ is normalized by the inverse interparticle spacing $\Delta^{-1}$. The neutral gas pressure was 19.3 $\mathrm{Pa}$ (upper row), 17.4 $\mathrm{Pa}$ (middle row), and $12 \mathrm{~Pa}$ (lower row). The left column is for the undisturbed crystal, the right column for the crystal with a single torsion (upper and middle row) or MCI (lower row). The discharge power $P_{\text {rf }}$ was as follows: (a) $7 \mathrm{~W}$, (b) $4 \mathrm{~W}$, (c) $20 \mathrm{~W}$, (d) $7 \mathrm{~W}$, (e) $20 \mathrm{~W}$, (f) $15 \mathrm{~W}$.

time domains, to arrive at the velocity power spectrum $V^{2}(k, f)$.

Results and discussion. - In the experimental runs employing argon pressures of $p_{\mathrm{Ar}}=19.3 \mathrm{~Pa}$ and $17.4 \mathrm{~Pa}$, torsions appeared at discharge powers as low as $P_{\mathrm{rf}}=4 \mathrm{~W}$ and $7 \mathrm{~W}$, respectively. As in Ref. [22], they were coupled pairs of apparently identical particles, oriented vertically and spun around their axis, see Fig. 1. The particle trajectories in a torsion are represented by a characteristic circle in the top view (left panel, center) and two parallel stripes in the side view (right panel, center). Reducing $P_{\text {rf }}$ even further resulted in more torsions joining the plasma crystal. Not all of them were performing clear circular motion. Some particles' orbits were ellipses with different degrees of elongation up to almost linear oscillations. A spinning pair could switch between different 


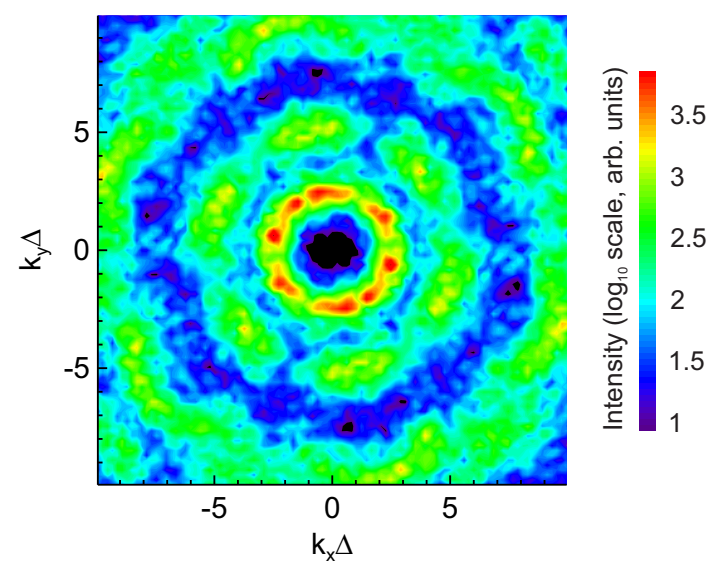

FIG. 4. Fluctuation spectrum of the particle velocity (compressional in-plane mode) integrated over the "hot-spot" frequency in the range of $20.6 \leq|f| \leq 23.6 \mathrm{~Hz}$ and shown as a function of $\left(k_{x}, k_{y}\right)$. The wave number is normalized by the inverse interparticle spacing $\Delta^{-1}$. The experimental parameters were as in Figs. 2 and 3(f).

regimes throughout its lifetime.

However, in the experimental run with $p_{\mathrm{Ar}}=12 \mathrm{~Pa}$, the torsion formation was preceded by the onset of the mode-coupling instability at $P_{\mathrm{rf}}=15 \mathrm{~W}$, see Fig. 2. Unlike in a torsion, many particles were involved in the MCI, since it is a collective effect. Here, the particle trajectories apparently have a circular shape in the vertical plane, as seen in the side view (right panel) [28]. Other MCI fingerprints, such as synchronization of the particle motion [29], were also observed. Further reduction of $P_{\text {rf }}$ led to eventual MCI-induced crystal melting. We note that in this experiment, MCI was observed at the highest neutral gas pressure so far.

Therefore, vertical pair formation and MCI are two competing scenarios for the crystal response to weakening vertical confinement. The neutral gas pressure seems to be a key factor in determining which process takes place first. These two cases are easily distinguished by the very different particle trajectories involved, compare Figs. 1 and 2 .

Once formed, a torsion becomes a source of disturbance in the plasma crystal. Through interaction with its neighbors, it redistributes a part of the energy which it receives from the flowing ions, to the surrounding lattice.

To study the effect of torsions on plasma crystal dynamics, we used fluctuation spectra of the particles' inplane velocities within the crystal. These are very sensitive to crystal parameters and details of the particle dynamics. In particular, the effect of MCI on the crystal spectra is well understood [16-18].

Amazingly, the spectra produced within a crystal by torsions and MCI have much in common and contain "hot spots" at similar locations on the $(k, f)$ plane [compare panels (b),(d) and (f) in Fig. 3], despite very different

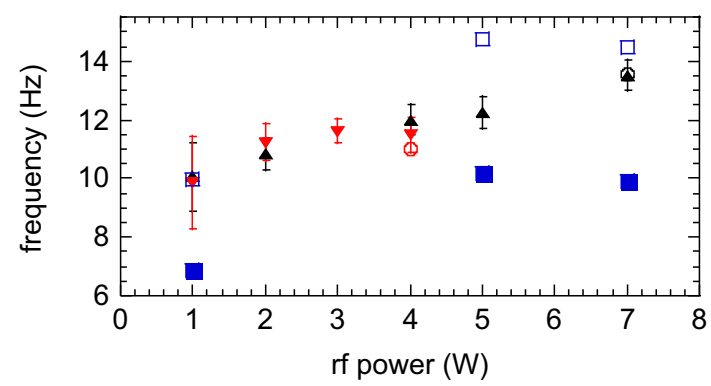

FIG. 5. "Hot-spot" frequency $f_{\mathrm{hs}}$ as a function of the discharge power $P_{\mathrm{rf}}$, for two different pressures of argon (up triangles are for $p_{\mathrm{Ar}}=17.4 \mathrm{~Pa}$, down triangles for $\left.19.3 \mathrm{~Pa}\right)$. Theoretically calculated (for $p_{\mathrm{Ar}}=17.4 \mathrm{~Pa}$ ) maximum frequency of the in-plane compressional mode $f_{\max }$ is shown by open squares and the lattice Einstein frequency $(2 \pi)^{-1} \omega_{\mathrm{E}}$ by solid squares. Where a single torsion was present, its rotation speed is shown by an open circle.

particle trajectories [compare Figs. 1 and 2]. This observation shows that torsions and MCI develop on the same spatial and temporal scales implying that deeper similarities between their governing mechanisms may also exist. To reveal the angular dependence of the particle velocity spectra, we used the method of Ref. [17] and plotted the spectra integrated over the "hot-spot" frequency as functions of $\left(k_{x}, k_{y}\right)$. The $\left(k_{x}, k_{y}\right)$ map for a crystal with MCI reveals a circular feature characteristic of the deep mode crossing [17], see Fig. 4. This probably explains why MCI developed at the relatively high pressure of $12 \mathrm{~Pa}$. We note that the MCI-induced hot spots in the crystal spectra such as Fig. 3(f) are a signature of the emergent unstable hybrid mode at the intersection of the out-ofplane and in-plane compressional crystal modes [17].

The frequency $f_{\mathrm{hs}}$ of the hot spots produced in the crystal spectrum by a torsion, see Figs. 3(b),(d), is closely related to the torsion rotation speed. We verified this by directly counting the number of turns a torsion makes per second in the original experimental video. $f_{\text {hs }}$ was measured by performing Gaussian fits of the peaks in the spectra averaged over the range of $k a=1.5-2.5$. The standard deviations of the fits are taken as error bars.

Upon further reduction of the discharge power at $p_{\mathrm{Ar}}=$ 19.3 $\mathrm{Pa}$ and $17.4 \mathrm{~Pa}$, multiple torsions appeared in the lattice. The crystal's fluctuation spectra, however, did not qualitatively change and remained similar to those in Figs. 3(b),(d). The hot-spot frequency $f_{\text {hs }}$ decreased as $P_{\mathrm{rf}}$ was reduced, as shown in Fig. $5 . f_{\text {hs }}$ turns out to be somewhat smaller than the maximum frequency of the in-plane compressional mode $f_{\max }$ yet larger than the lattice Einstein frequency $(2 \pi)^{-1} \omega_{\mathrm{E}}[30]$.

At the lowest discharge power used, $P_{\mathrm{rf}} \gtrsim 1 \mathrm{~W}$, almost all particles were arranged into vertical pairs. The amplitude of the particle displacements became small and it was no longer possible to reliably detect whether they were spinning or randomly oscillating. Multiple particle 


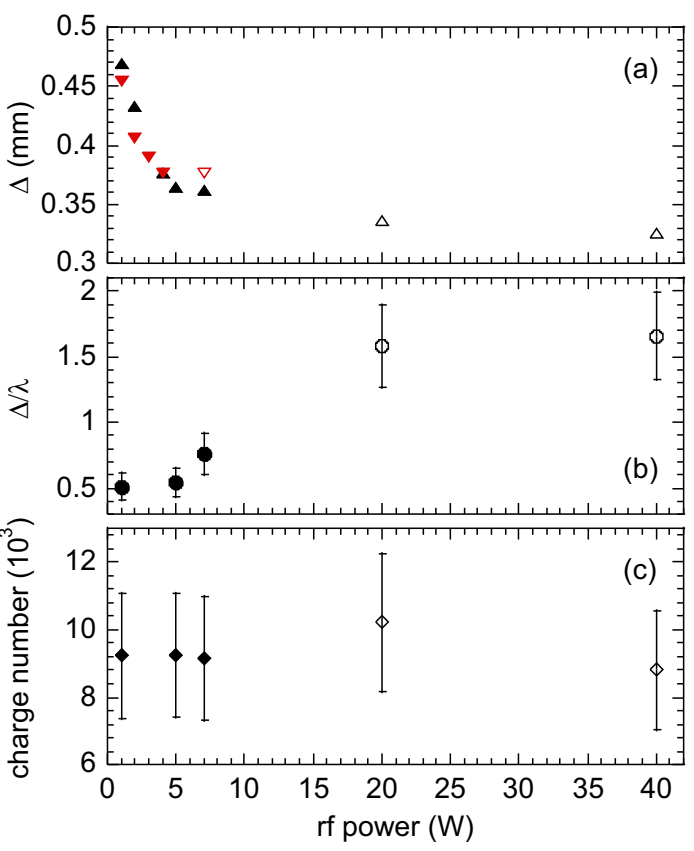

FIG. 6. Mean interparticle spacing $\Delta$ (a), screening parameter $\kappa=\Delta / \lambda(\mathrm{b})$, and particle charge number $Z$ (c) as functions of the discharge power $P_{\mathrm{rf}}$. Open symbols indicate undisturbed crystal, solid symbols indicate crystal with torsions. The pressure of argon was $p_{\mathrm{Ar}}=17.4 \mathrm{~Pa}$, except for down triangles in (a), which correspond to $p_{\mathrm{Ar}}=19.3 \mathrm{~Pa}$.

pairs formed a kind of disordered bilayer resembling the "pair state" reported in Ref. [33].

To shed light on the torsion formation mechanism, we studied how the main crystal parameters: mean interparticle spacing $\Delta$, screening parameter $\kappa \equiv \Delta / \lambda$ (where $\lambda$ is the screening length), and particle charge number $Z$, varied with discharge power. $\Delta$ was calculated as the position of the first peak in the pair correlation function for particles $g(r) . \quad Z$ and $\kappa$ were calculated using the standard method based on fitting the experimental fluctuation spectra of the particle velocities to the theoretical dispersion relations [31]. The results are shown in Fig. 6. At first glance, they seem to be rather counterintuitive: The torsions form when the mean interparticle spacing becomes large enough, see Fig. 6(a). At the same time, $\kappa$ becomes smaller, see Fig. 6(b). As a result, the screening length $\lambda=\Delta / \kappa$ grows even faster than $\Delta$, when reducing $P_{\text {rf }}$. This trend is consistent with the basic scalings: $\lambda \propto n_{i}^{-1 / 2}$ and $n_{i} \propto P_{\text {rf }}$ [34], where $n_{i}$ is the plasma density. The particle charge does not vary much with $P_{\mathrm{rf}}$, see Fig. 6(c).

We interpret these findings in the following way. Particle pairs form when the screening length of the screened Coulomb (Yukawa) interparticle interaction exceeds a threshold, i.e., when the interparticle interaction becomes sufficiently weakly screened. That allows more distant neighbors of a particle to exert a force on it. Therefore, the interaction of a particle with neighbors other than nearest, is probably essential for triggering the particle pairing. It is interesting to note that for lower gas pressures, the plasma crystal remains stable (neither particle pairing nor MCI develop) for larger screening lengths $($ smaller $\kappa)$. An open question is whether a "phase diagram" exists which delineates the regimes of a stable crystal, particle pairing, and MCI on the $\left(\kappa, \nu_{E}\right)$ plane, where $\nu_{E}$ is the neutral gas damping rate.

Finally, we note that a plasma crystal which contains multiple torsions in it is an interesting instance of a system of coupled rotators. As we showed in the present work, multiple torsions have rotation speeds that are distributed in a narrow range. Future work may include finding out whether there is phase synchronization between them [35].

Acknowledgments. - The authors thank Mierk Schwabe for helpful comments on the manuscript. V.N. gratefully acknowledges the hospitality experienced at Baylor University (USA).

*V.Nosenko@dlr.de

[1] A. Ivlev, H. Löwen, G. Morfill, C. P. Royall, Complex Plasmas and Colloidal Dispersions: Particle-resolved Studies of Classical Liquids and Solids, Series in Soft Condensed Matter Vol. 5 (World Scientific, Singapore, 2012).

[2] S. Nunomura, J. Goree, S. Hu, X. Wang, A. Bhattacharjee, K. Avinash, Phys. Rev. Lett. 89, 035001 (2002).

[3] A. Piel, V. Nosenko, J. Goree, Phys. Rev. Lett. 89, 085004 (2002).

[4] S. K. Zhdanov, S. Nunomura, D. Samsonov, and G. Morfill, Phys. Rev. E 68, 035401 (2003).

[5] K. Avinash, P. Zhu, V. Nosenko, and J. Goree, Phys. Rev. E 68, 046402 (2003).

[6] H. M. Thomas and G. E. Morfill, Nature (London) 379, 806 (1996).

[7] V. Nosenko, S. K. Zhdanov, A. V. Ivlev, C. A. Knapek, and G. E. Morfill, Phys. Rev. Lett. 103, 015001 (2009).

[8] A. Melzer, A. Schella, J. Schablinski, D. Block, and A. Piel, Phys. Rev. E 87, 033107 (2013).

[9] S. Nunomura, D. Samsonov, S. Zhdanov, and G. Morfill, Phys. Rev. Lett. 96, 015003 (2006).

[10] V. Nosenko and J. Goree, Phys. Rev. Lett. 93, 155004 (2004).

[11] A. Gavrikov, I. Shakhova, A. Ivanov, O. Petrov, N. Vorona, and V. Fortov, Phys. Lett. A 336, 378 (2005).

[12] P. Hartmann, M. C. Sándor, A. Kovács, and Z. Donkó, Phys. Rev. E 84, 016404 (2011).

[13] V. Nosenko, S. K. Zhdanov, A. V. Ivlev, G. E. Morfill, J. Goree, and A. Piel, Phys. Rev. Lett. 100, 025003 (2008).

[14] A. V. Ivlev, V. Nosenko, and T. B. Röcker, Contrib. Plasma Phys. 55, 35 (2015).

[15] A. V. Ivlev and G. Morfill, Phys. Rev. E 63, 016409 (2001).

[16] L. Couëdel, V. Nosenko, A. V. Ivlev, S. K. Zhdanov, H. M. Thomas, and G. E. Morfill, Phys. Rev. Lett. 104, 195001 (2010). 
[17] L. Couëdel, S. K. Zhdanov, A. V. Ivlev, V. Nosenko, H. M. Thomas, and G. E. Morfill, Phys. Plasmas 18, 083707 (2011).

[18] T. B. Röcker, A. V. Ivlev, S. K. Zhdanov, G. E. Morfill, Phys. Rev. E 89, 013104 (2014).

[19] C.-R. Du, V. Nosenko, S. Zhdanov, H. M. Thomas, and G. E. Morfill, Phys. Rev. E 89, 021101(R)(2014).

[20] S. K. Zhdanov, V. Nosenko, H. M. Thomas, G. E. Morfill, and L. Couëdel, Phys. Rev. E 89, 023103 (2013).

[21] S. K. Zhdanov, L. Couëdel, V. Nosenko, H. M. Thomas, and G. E. Morfill, Phys. Plasmas 22, 053703 (2015).

[22] V. Nosenko, S. K. Zhdanov, H. M. Thomas, J. CarmonaReyes, and T. W. Hyde, EPL 112, 45003 (2015).

[23] A. Melzer, V. A. Schweigert, I. V. Schweigert, A. Homann, S. Peters, and A. Piel, Phys. Rev. E 54, R46 (1996).

[24] V. A. Schweigert, I. V. Schweigert, A. Melzer, A. Homann, and A. Piel, Phys. Rev. E 54, 4155 (1996).

[25] C. M. Boessé, M. K. Henry, T. W. Hyde, and L. S. Matthews, Advances in Space Research 34, 2374 (2004).

[26] In many cases, torsions remained confined in the lattice cell where they nucleated, which facilitated recording their side-view video.
[27] U. Konopka, SPIT, Max-Planck-Institut für extraterrestrische Physik, D-85741 Garching, Germany / Auburn University, Auburn, AL, USA, 2005/2012.

[28] Elliptical trajectories were predicted by the theory of Ref. [18] and observed experimentally in Ref. [29].

[29] L. Couëdel, S. Zhdanov, V. Nosenko, A. V. Ivlev, H. M. Thomas, and G. E. Morfill, Phys. Rev. E 89, 053108 (2014).

[30] For a typical value of the coupling parameter $\kappa=1$, $2 \pi f_{\max } \approx 3.2 \omega_{0}[31]$ and $\omega_{\mathrm{E}} \approx 2 \omega_{0}$ [32], where $\omega_{0}=$ $\left(Q^{2} /\left(4 \pi \epsilon_{0} m \Delta^{3}\right)\right)^{1 / 2}$ and $m$ is the particle mass.

[31] S. Nunomura, J. Goree, S. Hu, X. Wang, A. Bhattacharjee, Phys. Rev. E 65, 066402 (2002).

[32] C. A. Knapek, A. V. Ivlev, B. A. Klumov, G. E. Morfill, and D. Samsonov, Phys. Rev. Lett. 98, 015001 (2007).

[33] V. Nosenko, A. V. Ivlev, R. Kompaneets, and G. Morfill, Phys. Plasmas 21, 113701 (2014).

[34] This scaling was roughly followed in an experiment with argon rf plasma at the pressure of $6 \mathrm{~Pa}$ using a similar setup. The plasma density was measured using microwave interferometry. L. Couëdel, private communication (2017).

[35] S. Olmi, E. A. Martens, S. Thutupalli, and A. Torcini, Phys. Rev. E 92, 030901(R) (2015). 